\title{
MBNIM: A MODULAR APPROACH TO FAST TRIGGER LOGIC FOR HIGH ENERGY PHYSICS EXPERIMENTS
}

\author{
A. BEER, F. BOURGEOIS, A. CORRE, G. CRITIN, M. L. HUBER, \\ R. PEGAITAZ, H. PFLUMM and G. SCHULER \\ CERN, Geneva, Switzerland
}

Received 14 August 1978 and in revised form 27 November 1978

\begin{abstract}
The Multi-Bit Nuclear Instruments Modules (MBNIM) have been developed for use in trigger logic for high energy physics experiments.

From a users point of view, the miniature coaxial cable is replaced by a 40 -wire flat cable bus carrying 16 counter signals thus significantly reducing the cost and the wiring effort.

A high degree of modularity has been achieved inside the units where standard front bus and CAMAC interlace boards are used.

Several NIM and CAMAC modules in the MBNIM standard are described together with examples of use.
\end{abstract}

\section{Introduction}

High energy physics experiments, in particular spectrometer experiments, may involve some or all of the following steps:

a) selection of events fulfilling some topological or other criteria that characterise the reaction one is looking for, by means of fast decision logic: the trigger logic;

b) on-line filtering of the selected interactions so that a "reasonable" - i.e. not too big amount of data is recorded on magnetic tape:

c) off-line pattern recognition and geometrical reconstruction of the data;

d) physics analysis.

It is well known that the third step, principally pattern recognition is at present the bottleneck as it requires a lot of computing power. Therefore, it is extremely important to achieve a good event selection in one of the first two steps. However, present standard Nuclear Instruments Modules are not very well suited for more complex trigger logic and physicists have to choose between:

1) fast, special-purpose, non-standard modules, or

2) refined filtering in the second step.

During the last two years a lot of effort has been put into the on-line filtering side using either minicomputers or microprocessors. At the same time it seems desirable that some effort be put in the field of fast decision logic, to minimize the data-rate at the beginning of the decision chain.

The Multi-Bit Nuclear Instrument Modules
(MBNIM) described in this paper have been developed for use around the $\Omega^{\prime}$ project at CERN.

The $\Omega$ spectrometer has been described in refs. $1-3$. The $\Omega^{\prime}$ project is the replacement of the present vertex detector - 15 spark chamber modules viewed by 8 computer controlled plumbicon cameras - by a set of multiwire proportional counters ( $\sim 40000$ sense wires). Various detectors are associated with the central vertex detector such as: scintillation and Cherenkov counter hodoscopes, drift chambers, $\gamma$ detectors etc. An electron beam and a radiofrequency separated hadron beam give the spectrometer a high degree of flexibility. Physicists from various European universities and laboratories use the spectrometer; it is the authors' duty to help them setting up their experiments (as well as servicing the detector) and, as a result of five years of operation the following remarks can be made:

1) Conventional NIM modules and miniature coaxial cables are no longer convenient to handle large arrays of counters, in that they require a lot of space, a lot of wiring and are consequently expensive.

2) Synchronization and gating requires a good knowledge of the system, which is not always the case for visiting teams.

3) Special purpose trigger logic often involves the same components (or functions) such as coincidence registers, level converters, memories, arithmetic and logical units etc.

4) However good the coordination may be, trigger modules designed outside the spectrome- 
ter environment often require additional hardware and software interfacing before they are ready to be used.

In fact, these remarks or some of them may be made in various high energy physics experiments as well as in the electronics service groups. Accordingly, the authors have tried to design a system of printed circuit boards and modules that would not only solve the above mentioned problems but also help the experimenter concentrate on his particular trigger problem.

\section{General description}

The Multi-Bit Nuclear Instruments Modules are characterised by the fact that they drive or read (counter) signals from a standard 40 -wire flat cable bus carrying 16 data lines, 2 synchronisation lines and one control line. In order to have clean and fast signals the bus is (1) driven by means of ECL $10 \mathrm{~K}$ gates of the type that is off in the low state (e.g. 10123 ), (2) terminated both ends.

Each signal line is separated from the neighbouring one by a ground line.

The connectors are 40 pin stamp-on socket connectors, SCOTCHFLEX type or equivalent. The cost of a one-meter cable equipped with connectors at both ends is five times lower than the solution involving 16 miniature coaxial cables. The 16 data lines may be used to carry bit patterns, coordinates, addresses or function codes.

The 2 synchronisation lines are optionally used by the modules for full handshake purposes. The

\section{WIRE N}
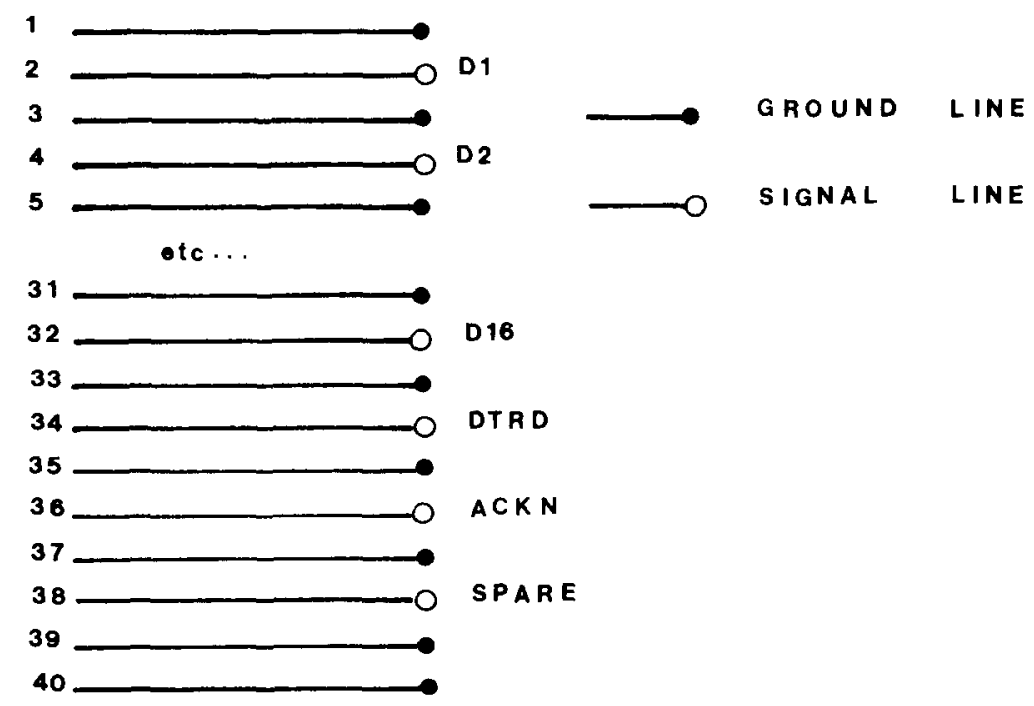

D 16

DT R D

ACK N

SPARE

Fig. 1. Flat cable bus: wire assignment. first one is the data ready line (DTRD), the second one is the acknowledge line (ACKN).

The control line is a spare data line that may be used to indicate read or write operations.

The wire assignments are shown in fig. 1 and the handshake timing diagram in fig. 2 .

It can be seen from fig. 2 that if more than two ports are connected to the same bus segment the DTRDs are wire-ored whilst the ACKNs are wireanded. Because of the very low output impedance of the ECL $10 \mathrm{~K}$ drivers, line ringing problems may be encountered when several driver modules are simultaneously forwarding data to two or more receivers. In this case, it is therefore necessary to have short bus segments between each driver module as well as between the receiver modules. Moreover, DTRDs must be synchronous and receiving modules must have the same timing properties.

Multiplexed transfers may occur on the bus. Module addressing and priority problems must however be solved by means of external wiring.

Though most modules are in the NIM or CAMAC standards, any other logic module may drive or read an MBNIM bus. However, in order to obviate the problems described in the introduction, standard interface boards have been designed:

1) the single-port interface which may also receive 8 miniature coaxial connectors giving access to 4 NIM to ECL $10 \mathrm{~K}$ level converters and $4 \mathrm{ECL} 10 \mathrm{~K}$ to NIM level converters (fig. 3);

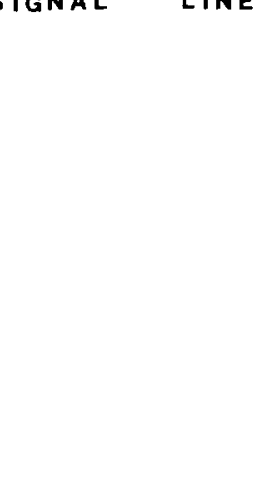




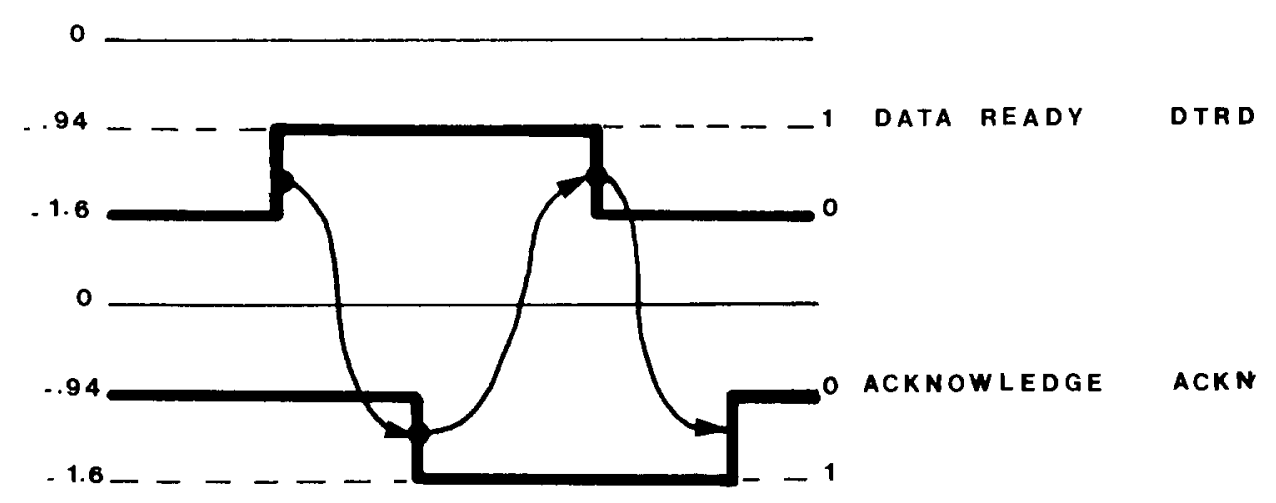

Fig. 2. Handshake - timing diagram.

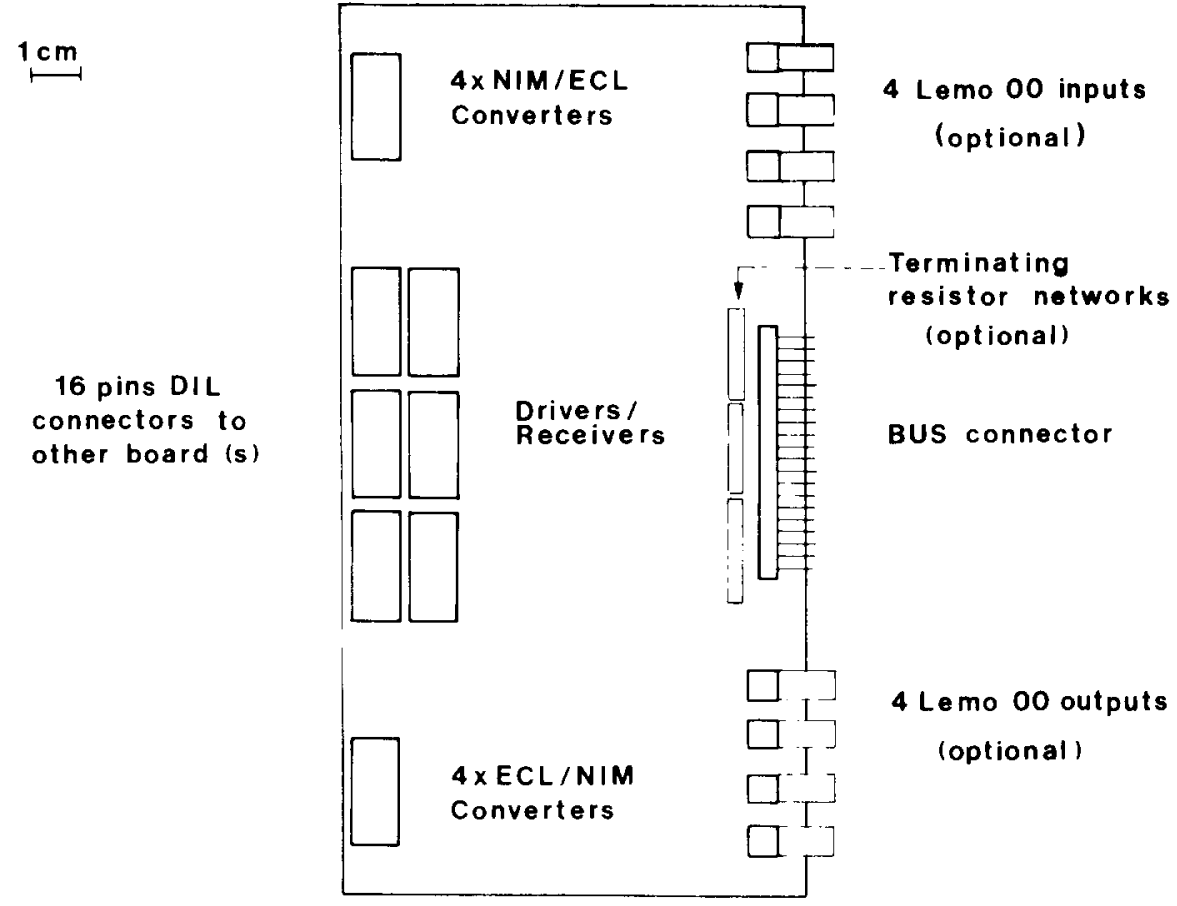

Fig. 3. Single-port interface board (schematic).

2) the dual-port interface (fig. 4).

These interface boards have been designed so that 10 ports can be connected on to the same bus. Three resistor networks can also be inserted to terminate the bus wherever necessary (a front panel light-emitting-diode indicates whether the termination is in or not).

At the rear of each interface port, two rows of three dual in line 16 pin connectors are used to transmit the bus signals to the function board in the user module. In this way the design of a spe- cial purpose board is simplified and data transmissions on the bus optimised. With one of each of the two interface boards, three port modules can be implemented in a standard single width NIM module (or double-width CAMAC module). The basic bus driver/receiver circuit is shown in fig. 5. The level converters available on the singleport interface board are shown in figs. $6 a$ and $b$.

In order to overcome the necessity of equalising time delays through busses and modules, most designs use the well known fast-clear technique, i.e. 


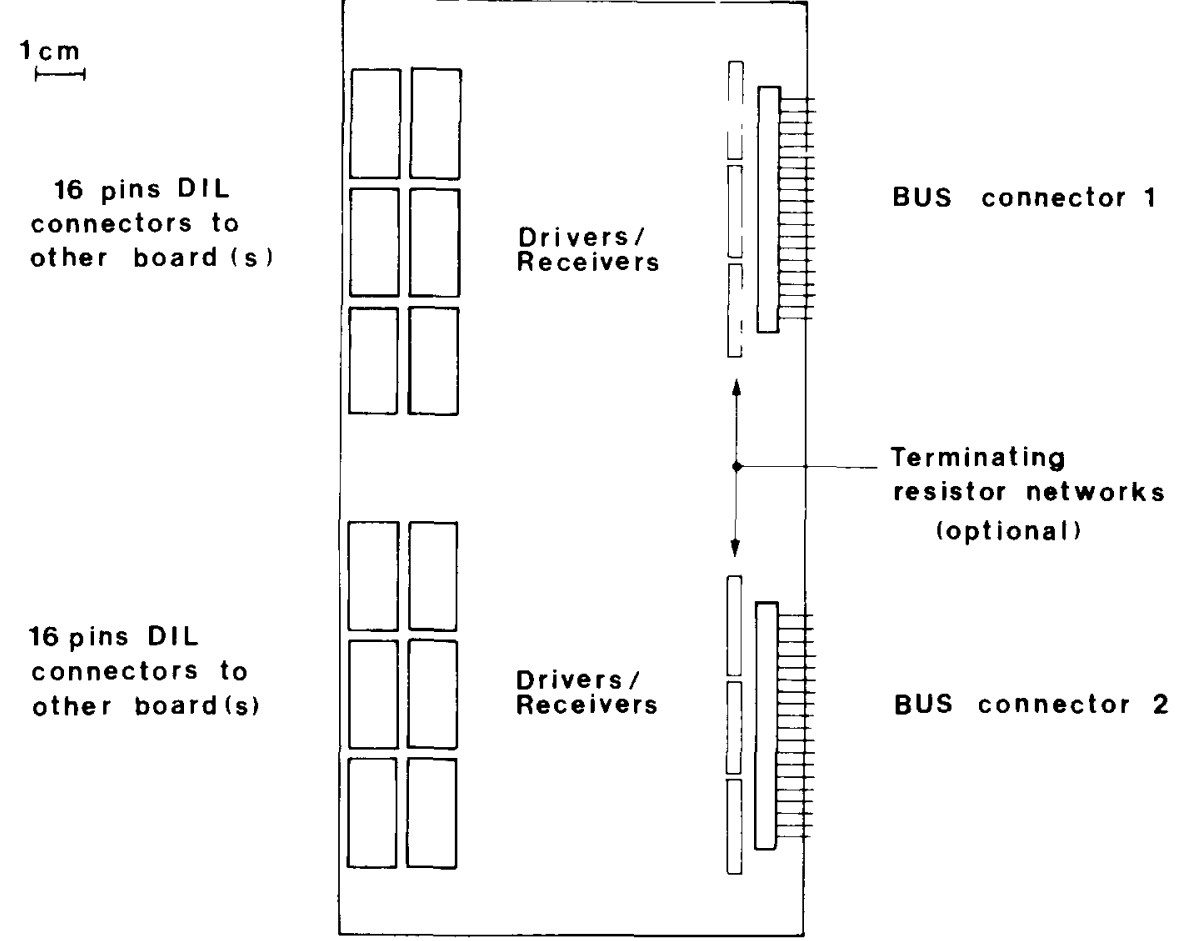

Fig. 4. Dual-port interface board (schematic).

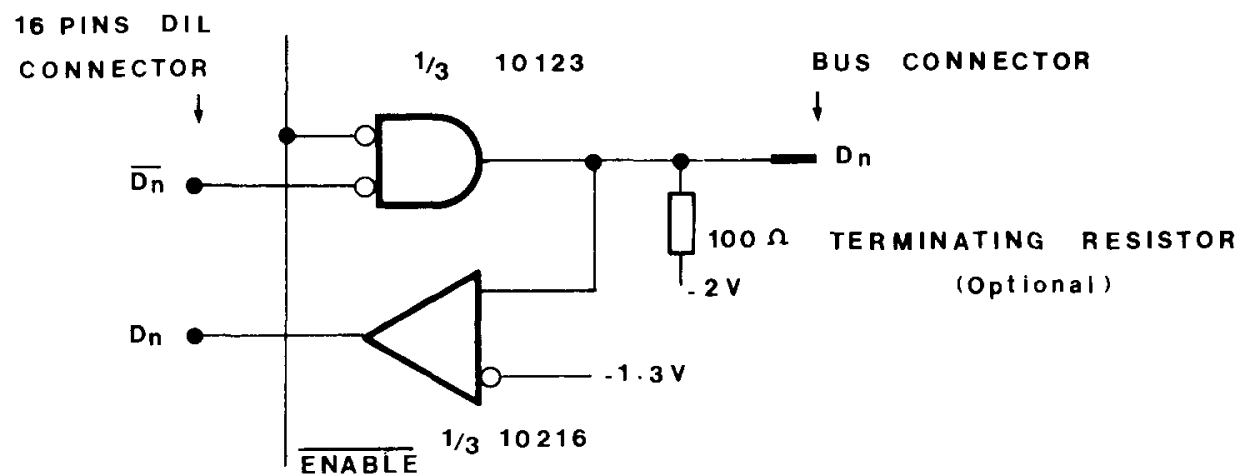

Fig. 5. Bus driver/receiver circuit.

data bits are sampled and latched into a register which is cleared once the processing is completed. However, by means of front panel wiring the user is free to choose between two solutions:

a) supply his own fast-clear and time it according to the overall time delay so that data input rate can be maximised,

b) use the built-in handshake mechanism so that he needs not care about timing problems.

The strobed coincidence register described below gives a good example of these two operating modes.
Standardisation has also been achieved on the CAMAC side of the modules which can be loaded with reference data (e.g. memory modules).

The standard CAMAC interface board is shown in fig. 7 ; it responds to the commands given in table 1 .

Three operating modes may be selected:

1) write data via CAMAC $(\mathrm{F}(24) \mathrm{A}(0))$,

2) check module contents $(F(24) A(1))$,

3) enable MBNIM bus operation $(\mathrm{F}(26) \mathrm{A}(0))$.

As the module memory size may exceed the CAMAC adressing capabilities, the write and check procedures are done in the well-known stop 


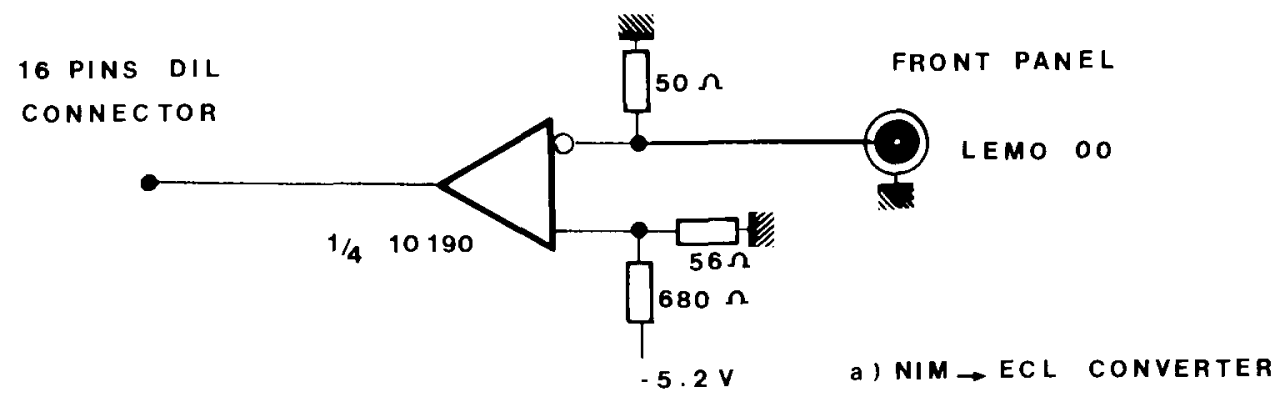

16 PINS DIL

CONNECTOR

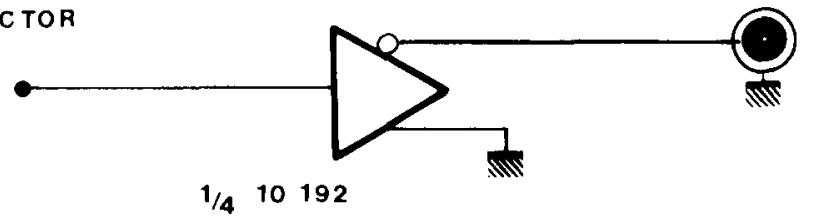

b) ECL $\rightarrow$ NIM CONVERTER

Fig. 6. (a) NIM to ECL IOK converter; (b) ECL $10 \mathrm{~K}$ to NIM converter.

mode ${ }^{4}$ ). However, in order to reduce the program size, the check procedure is arranged as follows:

a) a comparator is put on the memory board to compare the memory contents to the data that is presented on the write lines of the CAMAC dataway,

TABLE 1

List of commands.

\begin{tabular}{|c|c|c|c|}
\hline Command & $Q$ & $X$ & Comments \\
\hline$F(16) A(0)$ & yes & yes & $\begin{array}{l}\text { In write mode: write into mem- } \\
\text { ory at } \mathrm{S} 1 \text {, increment memory } \\
\text { address counter at } \mathrm{S} 2, Q=0 \text { af- } \\
\text { ter the last address has been } \\
\text { reached. } \\
\text { In check mode: compare data to } \\
\text { memory contents at } \mathrm{S} 1 \text {, incre- } \\
\text { ment address counter at } \mathrm{S} 2 \text {. } \\
Q=0 \text { if error condition is en- } \\
\text { countered. }\end{array}$ \\
\hline$F(24) A(0) S 2$ & no & yes & $\begin{array}{l}\text { Clear memory address register, } \\
\text { set to write mode. Disable front } \\
\text { bus operation. }\end{array}$ \\
\hline$F(24) A(1) S 2$ & no & yes & $\begin{array}{l}\text { Clear memory address register, } \\
\text { set to check mode. Disable } \\
\text { front bus operation. }\end{array}$ \\
\hline$F(26) A(0) S 2$ & no & yes & Enable front bus operation. \\
\hline $\mathrm{F}(27) \mathrm{A}(0)$ & yes & yes & Check error flag. \\
\hline
\end{tabular}

b) during the actual check procedure, data is written again into the module; whenever a mismatch is found by the comparator, further $Q$ responses are inhibited and an error flag is set.

It is readily seen that, in this way, the program size is significantly reduced with respect to the conventional solution where memory contents are read back into the host computer and compared to the reference data.

\section{Some examples of MBNIM modules}

\subsection{THE STROBED COINCIDENCE REGISTER}

This module is used to strobe, latch and convert to the bus standard up to 16 NIM pulses e.g. conventional discriminator outputs. However, in order to have a short resolving time, the coincidence is not of the conventional overlap type. As shown in fig. 8 , an output bit is set to logical-1 by a negative-going transition on the data input (NIM) if, and only if this transition occurs during the strobe. Data remains stable until a fast clear (NIM) is applied at the clear input.

At the end of the strobe, a BUSY signal (NIM) is generated which remains until the end of the clear.

Moreover, the MBNIM bus acknowledge ACKN (NIM) is supplied on the front panel (fig. 9) and 


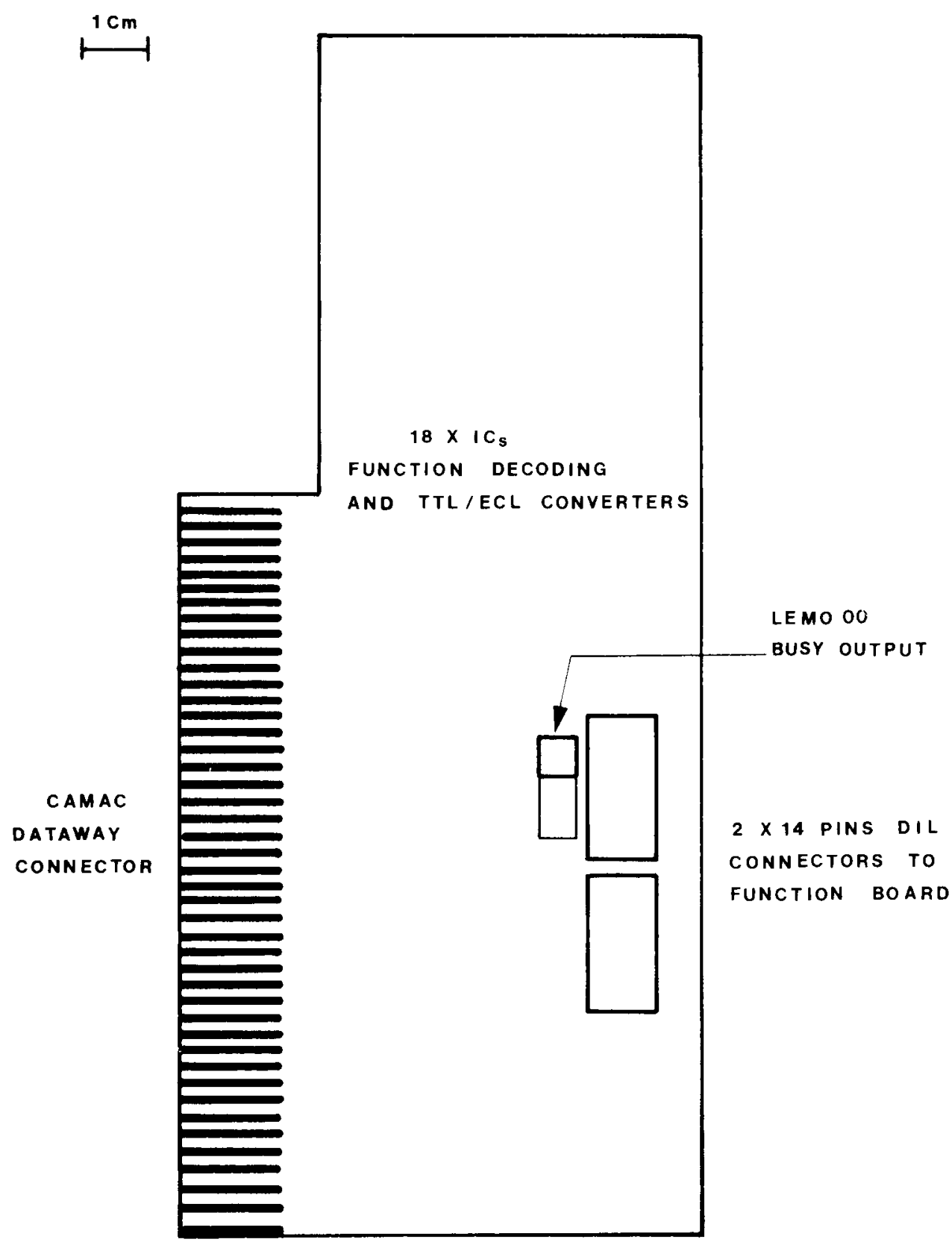

Fig. 7. CAMAC interface board for memory modules (schematic).

may be used as a clear thus making the bus operate in full handshake mode.

The data input rate has a minimum guaranteed value of $170 \mathrm{MHz}$ and the dead time (BUSY time) of the module in full handshake mode may be as low as $80 \mathrm{~ns}$.

The module has been designed using the singleport interface and it is normally equipped with ter- minating resistors which may however be removed if the module is in the middle of a bus section.

The module fits into a standard NIM crate and requires only the $-6 \mathrm{~V}$ power supply.

3.2. The TERMinim module

This module is used to convert bus signals to 


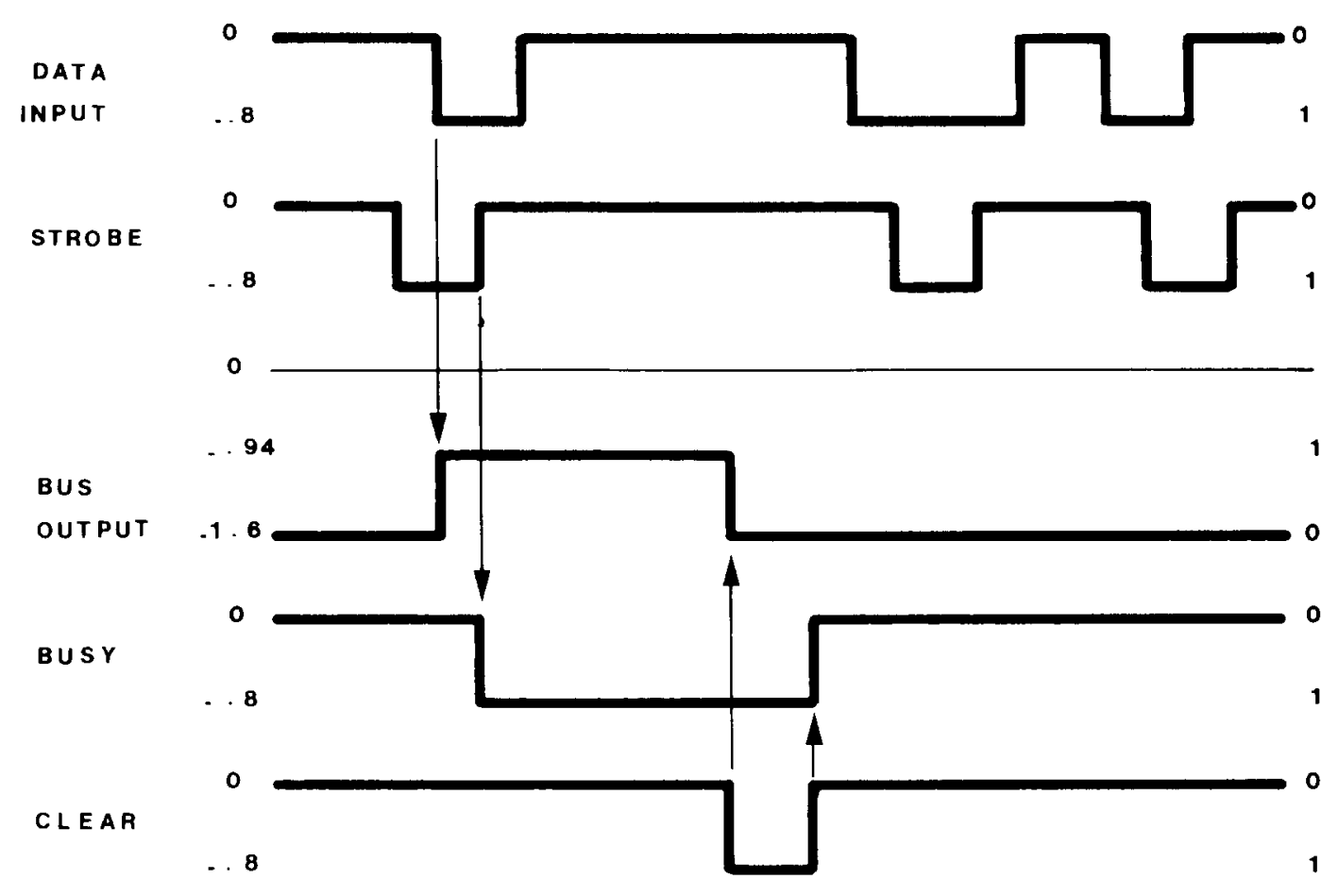

Fig. 8. Strobed coincidence register: timing diagram.

NIM levels. The data ready output DTRD may be fed into the ACKNowledge input thus giving full handshake operation on the bus section.

The module has been designed using the singleport interface and it is normally equipped with terminating resistors. It fits into a standard NIM crate and requires only the $-6 \mathrm{~V}$ power supply.

\subsection{THE BIT ASSIGNER}

This CAMAC module has been designed using the dual port front bus interface and the CAMAC board described in the second paragraph.

To each bit of the input bus, the BIT ASSIGNER assigns logical-1 values to sets of bits of the output bus. When several bits are at logical-1 value in the input bus, the bit to bit OR of the assigned patterns is presented on the output bus.

A straightfoward application of this module is in the construction of coincidence matrices. Fig. 10 shows a typical high energy physics trigger set-up: $\mathrm{H} 1$ and $\mathrm{H} 2$ are two hodoscopes (of 16 elements each for simplicity) and $T$ is the target in which beam particles interact. In order to select events, one must check whether a hit in $\mathrm{Hl}$ has been produced by a particle coming from the target. It is readily seen that to each element of $\mathrm{Hl}$ corresponds one or more elements of $\mathrm{H} 2$. For a given pattern in $\mathrm{H} 1$, the BIT ASSIGNER will give the predicted pattern in $\mathrm{H} 2$. With other MBNIM modules the actual pattern in $\mathrm{H} 2$ may be compared to the predicted pattern generated by the BIT ASSIGNER. For this purpose, an MBNIM bit to-bit AND module has been designed, which also gives information on the number of bits set in the AND pattern. A schematic of a $16 \times 16$ coïncidence matrix using the above modules is given in fig. 11 .

The BIT ASSIGNER may also be used to generate 16 OR functions of 16 variables; it has a 40 ns input to output propagation delay.

The function board of the BIT ASSIGNER uses 16 fast $16 \times 4$ bit ECL random-access memories (10 145) which are assembled in 4 rows of $16 \times 16$ bit words. Assigned patterns must be generated per group of 4 bits and therefore 64 patterns must be written via CAMAC before the module can be used. A BUSY signal (NIM) indicates whether the module is ready for use. 


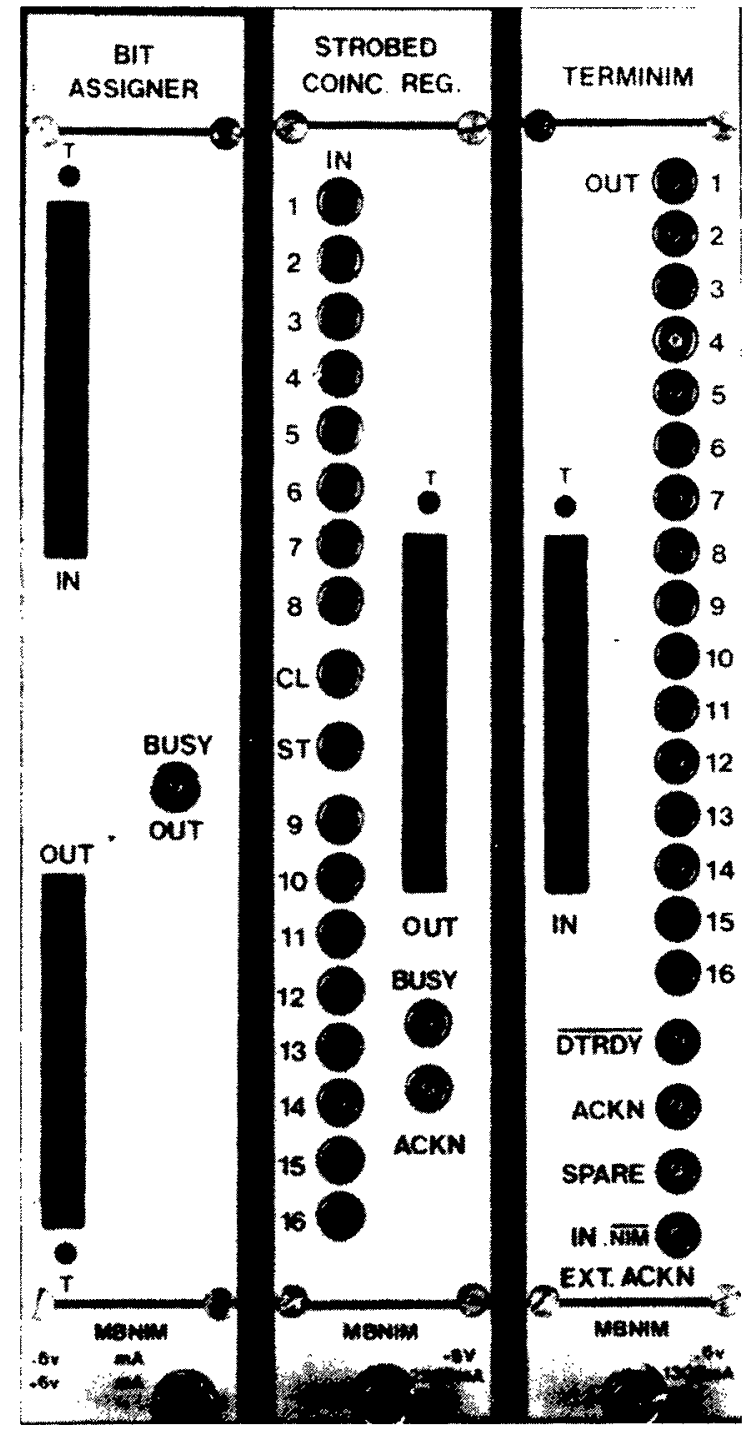

Fig. 9. Some MBNIM modules (front panels).

\subsection{Other MBNiM mOdules}

Several modules have been designed such as the AND module mentioned in the previous section. which may be used as an OR or an exclusive OR by changing 4 ICs on the function board. Other modules are currently under design:

a) a $1024 \times 16$ bit MEMORY with address input and data input/output; the module is controlled via CAMAC with the standard CAMAC interface card described in the second section,

b) an ECL arithmetic and logical unit to process the multiplicity information supplied by two or more AND modules,

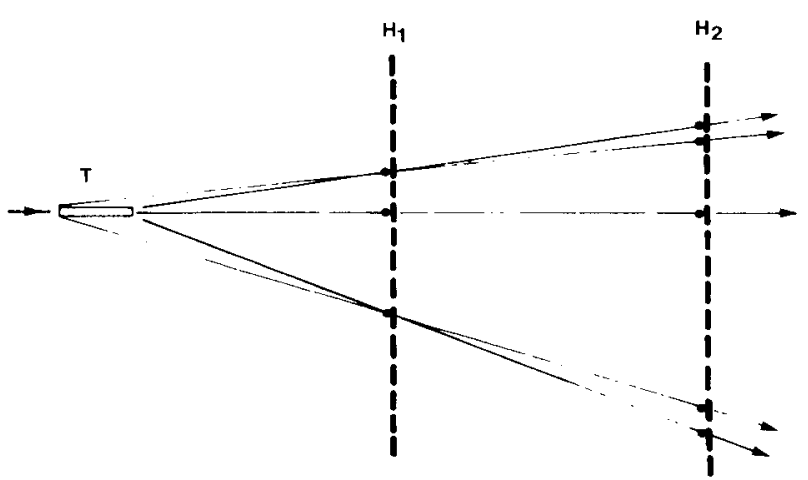

Fig. 10. Typical hodoscope arrangement in an electronics experiment.

c) a 4 lines to 16 lines DECODER to convert the binary multiplicity into fully decoded NIM signals.

\section{Conclusion}

The high degree of modularity achieved in the MBNIM standard has proved to be extremely useful during the design phase as the development was carried out while the authors were spending most of their time servicing the present Omega spectrometer. It is hoped that the system will expand inside and outside CERN as it solves most of the limitations of standard NIM modules described overleaf.

Complex sequential trigger logic may also be wired combining conventional NIM modules with MBNIM boxes. For example, one may build the 64-bit hash addressing logic described in ref. 5 with very little extra, special purpose hardware.

Finally, the modules described in this paper are easy to wire and therefore fairly cheap.

The authors would like to thank Drs. W. Beusch, O. Gildemeister and P. Sonderegger for their support in this work. They also acknowledge most valuable advice from Messrs. T. Armstrong and C. Bizeau.

\section{References}

1) A. Michelini. Summer study on AGS urilization (Brookhaven National Laboratory, 1970) p. 279.

2) F. Bourgeois and A. Corre. Nucl. Instr. and Meth. 108 (1973) 485.

3) W. Beusch, CERN/SPSC 77-70 (1977).

4) Esone Committee. EUR 4100e (Euratom, Luxembourg, August 1972).

5) A. de Bellefon et al., CERN/EF 77-1 (1977). 
4 X CONVENTIONAL

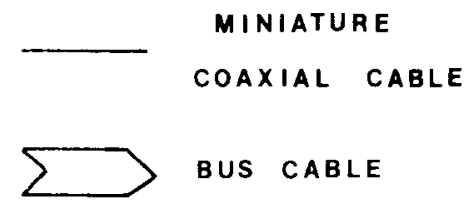

DISCRMINATORS

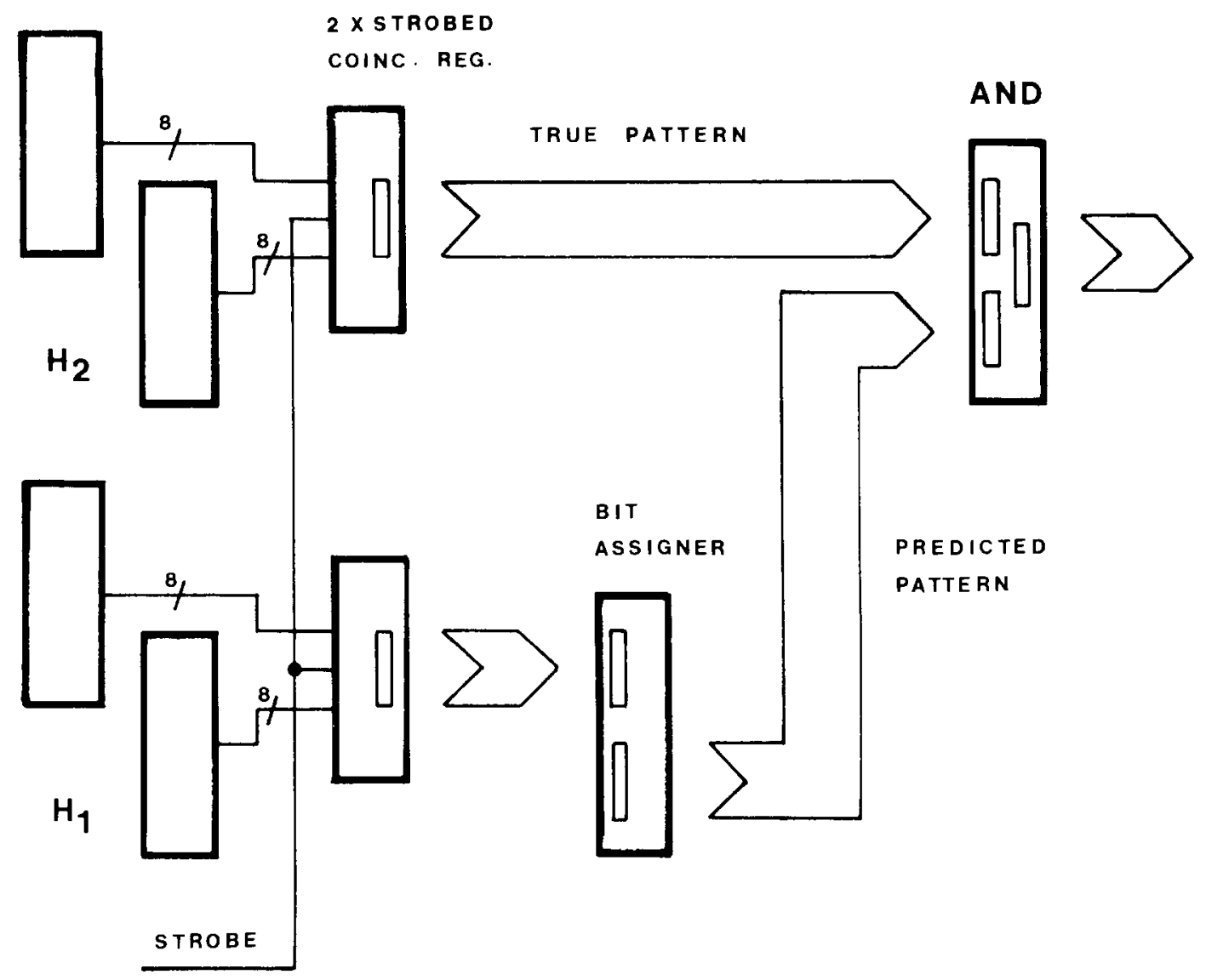

Fig. 11. Schematic of a $16 \times 16$ coincidence matrix using MBNIM modules. 